\title{
Corner diffraction coefficients for the quarter plane
}

\author{
Hansen, Thorkild B.
}

Published in:

I E E E Transactions on Antennas and Propagation

Link to article, DOI:

10.1109/8.86918

Publication date:

1991

Document Version

Publisher's PDF, also known as Version of record

Link back to DTU Orbit

Citation (APA):

Hansen, T. B. (1991). Corner diffraction coefficients for the quarter plane. I E E E Transactions on Antennas and Propagation, 39(7), 976-984. https://doi.org/10.1109/8.86918

\section{General rights}

Copyright and moral rights for the publications made accessible in the public portal are retained by the authors and/or other copyright owners and it is a condition of accessing publications that users recognise and abide by the legal requirements associated with these rights.

- Users may download and print one copy of any publication from the public portal for the purpose of private study or research.

- You may not further distribute the material or use it for any profit-making activity or commercial gain

- You may freely distribute the URL identifying the publication in the public portal

If you believe that this document breaches copyright please contact us providing details, and we will remove access to the work immediately and investigate your claim. 


\title{
Corner Diffraction Coefficients for the Quarter Plane
}

\author{
Thorkild B. Hansen, Student Member, IEEE
}

\begin{abstract}
The current near a right-angled corner on a perfectly conducting flat scatterer illuminated by a plane wave is expressed as a sum of three currents. The first is the physical optics (PO) current, which describes the surface effect. The second is the fringe wave (FW) current, which is found from the half-plane solution and accounts for the distortion of the current caused by the edges. The third is the corner current, which is found from the numerical solution to the electric-field integral equation applied to the square plate, and accounts for the distortion of the current caused by the corner. It is found that the corner current for the right-angled corner, illuminated from a forward direction, consists mainly of two edge waves propagating along the edges forming the corner. Analytical expressions for these edge wave currents are constructed from the numerical results. A corner diffracted field is calculated by evaluating the asymptotic corner contributions to the radiation integral over the sum of the three currents. It is found that the corner contribution from the edge wave currents in some cases is of the same size as the corner contributions from the physical optics current and the fringe wave current.
\end{abstract}

\section{INTRODUCTION}

$\mathrm{T}$ HE only canonical scattering problem for the calculation of corner diffraction that has been solved is the problem of scattering from the perfectly conducting plane angular sector. A dyadic Green's function for this problem was first derived by Satterwhite [1] using the method of separation of variables in spheroconal coordinates. An alternative derivation is given in [2]. This dyadic function is in a complicated nonclosed-form expression involving spherical Bessel and Lamé functions. It has very poor convergence properties when both source and field points are far from the corner [3]. Therefore, it is not a useful solution for high frequency corner diffraction calculations. By using the characteristic Green's function method [4, sec. 3.3] an attempt has been made to obtain a representation which is useful for high frequency diffraction [5]. However, because the separation constants in the solution to the Helmholtz equation in spheroconal coordinates are coupled $[6$, p. 518], this attempt was not successful. Recently, Smyshlyaev [7]-[9] obtained expressions for the field diffracted by the corner on a plane angular sector. However, these expressions are also in a very complicated nonclosed form difficult to use in numerical evaluations. Therefore, it seems that much work still has to be done in order to obtain a useful corner diffraction formula

Manuscript received July 27, 1990; revised January 30, 1991. The author is with the Electromagnetics Institute, Technical University of Denmark, DK-2800 Lyngby, Denmark.

IEEE Log Number 9100568.

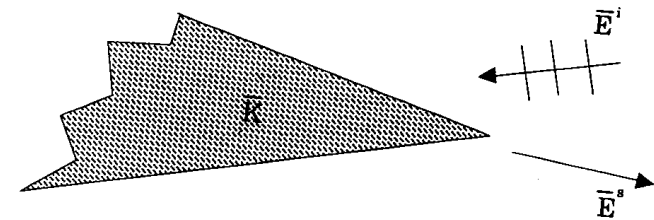

Fig. 1. Scattering configuration

based on the exact eigenfunction solution to a conical scattering problem.

In this paper we will obtain a corner diffraction coefficient based on an exact numerical solution to a canonical scattering problem. To explain the approach that we use, consider the scattering configuration shown in Fig. 1.

An infinitesimally thin, perfectly conducting scatterer is illuminated by the incident plane-wave field $\bar{E}^{i}$. The incident field induces a surface current density $\bar{K}$ on the scatterer that radiates the scattered field $\bar{E}^{s}$. We will find an approximation to the current $\bar{K}$ and then calculate the corner diffracted field by evaluating the asymptotic corner contribution to the radiation integral over $\bar{K}$. The current can be written as

$$
\bar{K}=\bar{K}^{\mathrm{PO}}+\bar{K}^{\mathrm{FW}}+\bar{K}^{c}
$$

where these three components of current on the right-hand side of (1) accounts for three different diffraction phenomena on the scatterer. $\bar{K}^{\mathrm{PO}}$ is the physical optics current, which describes the surface effect, $\bar{K}^{\mathrm{FW}}$ is the fringe wave current, which describes the distortion caused by edges, and $\bar{K}^{c}$ is the corner current, which describes the distortion caused by the corner.

The fringe wave current is found from the half-plane solution. An approximation to the corner current will be found, for forward directions of incidence, for the right-angled corner by solving numerically the electric-field integral equation applied to a square plate. From these numerical results we will construct approximate analytical expressions for this current for a wide range of angles of forward incidence. Finally, we will determine whether the corner current can be important in the calculation of the corner diffracted field. Throughout this paper we assume $e^{j \omega t}$ time harmonic fields.

\section{Physical Optics and Fringe Wave Currents}

This section deals with the physical optics current and the fringe wave current for the scattering problem shown in Fig. 1. The physical optics current is given by

$$
\bar{K}^{\mathrm{PO}}=2 \hat{n} \times \bar{H}^{i}
$$




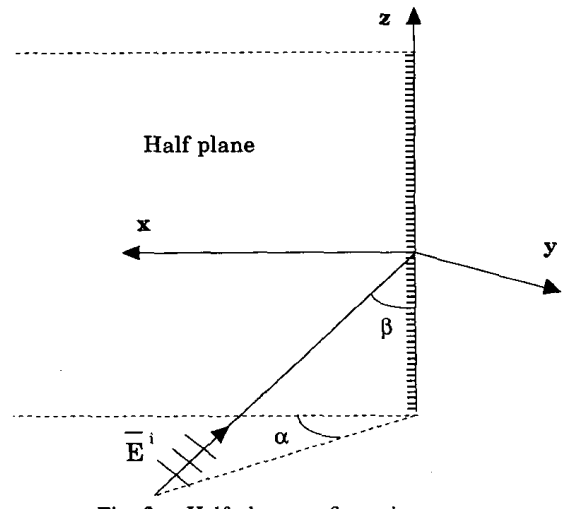

Fig. 2. Half-plane configuration.

where $\hat{n}$ is a normal unit vector to the scatterer and $\bar{H}^{i}$ is the incident magnetic field. The physical optics current is often referred to as the tangent-plane approximation since it is obtained by assuming that the current at each point of the scatterer is the current that would exist on an infinite plane, tangent to the surface of the scatterer at that point. The physical optics current does not take into account the distortion of the current caused by edges and corners. The current that accounts for the distortion caused by the edges is the fringe wave current denoted by $\bar{K}^{\mathrm{FW}}$. In order to explain how this current is obtained consider the half-plane problem shown in Fig. 2. The half-plane is given by $y=0, x \geq 0$ and the direction of incidence is given by the angles $\alpha$ and $\beta$ as shown. The incident plane-wave field is given by

$$
\bar{E}^{i}(\bar{r})=\bar{E}_{0}^{i} e^{-j \bar{k} \cdot \bar{r}}
$$

and

$$
\bar{H}^{i}(\bar{r})=\vec{H}_{0}^{i} e^{-j \bar{k} \cdot \bar{r}}
$$

where the propagation vector is

$$
\bar{k}=-k(\hat{x} \sin \beta \cos \alpha+\hat{y} \sin \beta \sin \alpha-\hat{z} \cos \beta) .
$$

$\bar{E}_{0}^{i}$ and $\bar{H}_{0}^{i}$ are the constant amplitudes of the electric and magnetic fields, and $k$ denotes the free-space propagation constant. The half-plane fringe wave (HPFW) current $\widetilde{K}^{\mathrm{HPFW}}$ is defined by

$$
\bar{K}^{\mathrm{HPFW}}(x, z)=\bar{K}^{\mathrm{HP}}(x, z)-\bar{K}^{\mathrm{PO}}(x, z)
$$

where $\bar{K}^{\text {HP }}$ is the total half-plane current found from the exact half-plane solution, and $\bar{K}^{\mathrm{PO}}$ is the physical optics current on the half-plane. The half-plane fringe wave current can be found from [10, ch. 8], and is given explicitly in [11]. The $x$ and $z$ components of this current are given by

$$
\begin{array}{r}
K_{x}^{\mathrm{HPFW}}(x, z)=-\frac{4 e^{j \frac{\pi}{4}}}{\sqrt{\pi}} \bar{H}_{0}^{i} \cdot \hat{z} F\left(\sqrt{2 k x \sin \beta} \cos \frac{\alpha}{2}\right) \\
\cdot e^{j k(x \sin \beta \cos \alpha-z \cos \beta)}
\end{array}
$$

and

$$
\begin{aligned}
K_{z}^{\mathrm{HPFW}} & (x, z) \\
= & \frac{4 e^{j \frac{\pi}{4}}}{\sqrt{\pi}} \frac{1}{\sin \beta} \\
& \cdot\left[\left\{\bar{H}_{0}^{i} \cdot \hat{z} \cos \beta \cos \alpha-\frac{1}{\zeta} \bar{E}_{0}^{i} \cdot \hat{z} \sin \alpha\right\}\right. \\
& \cdot F\left(\sqrt{2 k x \sin \beta} \cos \frac{\alpha}{2}\right) e^{j k(x \sin \beta \cos \alpha-z \cos \beta)} \\
+ & \left\{-\bar{H}_{0}^{i} \cdot \hat{z} \cos \frac{\alpha}{2} \cos \beta+\frac{1}{\zeta} \bar{E}_{0}^{i} \cdot \hat{z} \sin \frac{\alpha}{2}\right\} \\
& \left.\cdot \frac{e^{-j k(x \sin \beta+z \cos \beta)}}{j \sqrt{2 k x} \sin \bar{\beta}}\right]
\end{aligned}
$$

where $\zeta$ is the free-space impedance and $F(x)$ is the Fresnel function defined by

$$
F(x)=\int_{x}^{+\infty} e^{-j t^{2}} d t .
$$

To study the half-plane fringe wave current far from the edge we use the asymptotic value of the Fresnel function

$$
F(x) \sim \frac{-j}{2 x} e^{-j x^{2}}, \quad \text { as } x \rightarrow+\infty
$$

in the current expressions (7) and (8) and find

$$
\begin{aligned}
\bar{K}^{\mathrm{HPFW}}(x, z) \sim & \frac{-2 e^{-j \frac{\pi}{4}} H_{z}^{i}\left(0,0, z^{\prime}\right)}{\sin ^{2} \beta \cos \frac{\alpha}{2} \sqrt{2 \pi k l}} \\
& \cdot[\hat{x} \sin \beta+\hat{z} \cos \beta] e^{-j k l}, \\
& \text { as } \sqrt{2 k l} \sin \beta \cos \frac{\alpha}{2} \rightarrow+\infty
\end{aligned}
$$

where the distances $z^{\prime}$ and $l$ are defined in Fig. 3. We see that the half-plane fringe wave current at $(x, z)$ is launched by the incident field at the edge point $z^{\prime}$ and that it propagates and points in the direction of the Keller cone. Furthermore, except at grazing incidence it is a current that tends to zero as the distance $l$ from the edge gets large. The fringe wave current $\bar{K}^{\mathrm{FW}}$ on the original scatterer is simply the sum of the half-plane fringe wave currents that are launched at the edges. This current is said to be caused by first-order edge diffraction and it accounts for the distortion of the current caused by the edges. Since the fringe wave current is found from currents that are launched at the edge of a half-plane it does not take into account the distortion of the current caused by the corner.

\section{Corner Currents}

The current that accounts for the distortion caused by the corner, denoted by $\bar{K}^{c}$ in (1), will be found approximately by using the method of moments solution to the electric-field integral equation for the square plate. The version of the moment method that we use here was developed by Wilton and Glisson [12] and the matrix equation is solved using the 


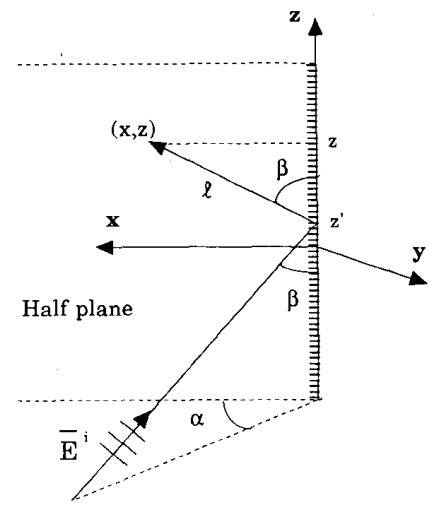

Fig. 3. Propagation properties of half-plane fringe wave currents.

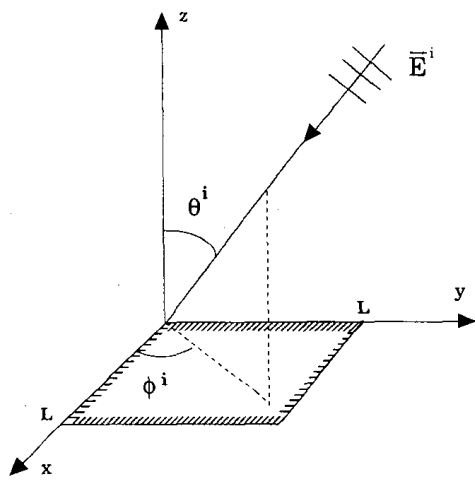

Fig. 4. Square plate configuration

conjugate gradient method [13]. This moment method calculates the mean values of current components on square patches on the plate.

To get information about $\bar{K}^{c}$ we will investigate the current obtained by subtracting the physical optics current and the fringe wave currents from the moment method (MM) current. The current obtained in this manner will be called the MM-PO-FW current. In order to find $\bar{K}^{c}$ from the MM-PO-FW current we have to investigate some of the diffraction processes that occur on the square plate. Let the square plate be situated in the $x-y$ plane of a rectangular coordinate system as shown in Fig. 4. The side length of the plate is denoted by $L$ and the direction of incidence of the plane wave is determined by the spherical coordinates $\theta^{i}$ and $\phi^{i}$ as shown. Let us consider the case where the direction of incidence is given by $\left(\theta^{i}, \phi^{i}\right)=\left(45^{\circ}, 45^{\circ}\right)$. In this case the propagation direction of the fringe wave currents emanating from the edge on the $x$-axis form an angle of $60^{\circ}$ with the negative $x$-axis as shown in Fig. 5 . We see that these fringe wave currents introduce normal components of current on the part of the circumference marked by $\mid$. On this part of the circumference diffraction processes occur and new currents are launched. These new currents are said to be caused by second-order edge diffraction. By investigating the propagation directions of the fringe wave currents from all the edges one finds that no second-order edge diffracted current is launched on the part of the circumference, that is marked by

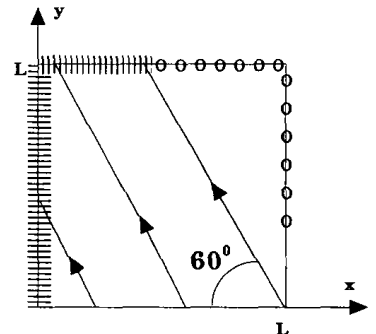

Fig. 5. Propagation directions of fringe wave currents emanating from the edge on the $x$-axis. Region where these fringe wave currents launches second-order currents. 0 Region where no second-order currents are launched.

0 in Fig. 5 [14]. From Fig. 5 it is seen that when $\left(\theta^{i}, \phi^{i}\right)=$ $\left(45^{\circ}, 45^{\circ}\right)$ the corner at $x=y=L$ is the only corner at which no second-order currents are launched. Since we do not have general expressions for second-order currents we can only determine the corner current from the MM-PO-FW current at this corner. One finds that this is always the case when $0<\phi^{i}<90^{\circ}$.

Since the moment method calculates mean values of current components on square patches we also have to calculate mean values of the physical optics current and the fringe wave currents before we can evaluate the MM-PO-FW current. The physical optics current has no singularities and does not vary much on a patch when the side length of the patch is much smaller than a wavelength. Therefore, the mean value of the physical optics current on a patch can be approximated by the center value. This is also the case for the fringe wave current except near the edges where it has a singularity (8). At the edges we divide the patches into smaller rectangles which are parallel to the edge of the square plate and calculate center values of the fringe wave currents on these smaller rectangles. The mean values of the fringe wave currents on the patches are then found by adding the center values on the smaller rectangles and dividing this by the number of rectangles.

\section{Numerical Results for MM-PO-FW CuRRENTS}

We will now show numerical results for the MM-PO-FW current. The configuration is shown in Fig. 4 and the direction of incidence is given by $\left(\theta^{i}, \phi^{i}\right)=\left(45^{\circ}, 45^{\circ}\right)$. The incident plane wave is polarized in the theta direction and it is normalized so that $\left|\bar{H}^{i}\right|=1$. In this case the current is symmetric about the line $x=y$, i.e., $K_{x}(x, y)=K_{y}(y, x)$ so we will only show the $x$-component. The side length of the square patches used in the moment method program is $1 / 8 \lambda$ where $\lambda$ is the wavelength. Figs. 6 and 7 show the amplitude of the MM-PO-FW currents for the $4 \lambda$ and $7 \lambda$ plate, respectively. Before we discuss the results we note that there, in general, will be discontinuities in the MM-PO-FW current at the shadow boundaries of the fringe wave current. However, for the case considered in Figs. 6 and $7\left(\theta^{i}, \phi^{i}=\right.$ $45^{\circ}$ ) these discontinuities are very small.

Let us first consider the $4 \lambda$ results. We see that the current on the interior part of the plate is small compared to physical optics and that there are some large currents along the edge. 


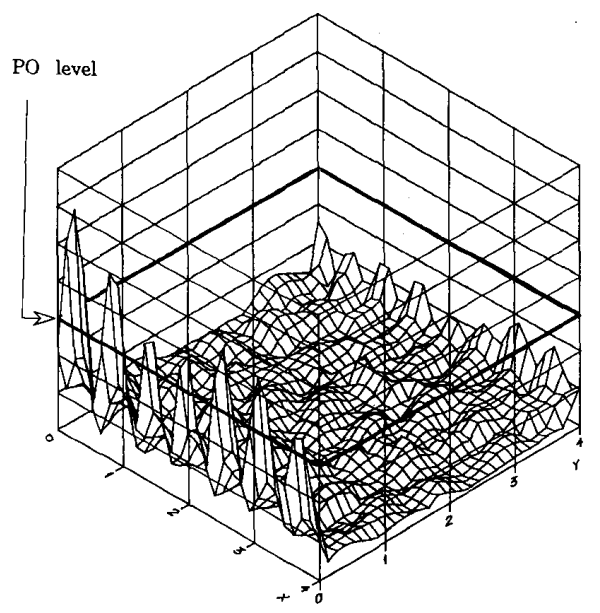

Fig. 6. $x$-component of MM-PO-FW current. Direction of incidence: $\left(\theta^{i}, \phi^{i}\right)=\left(45^{\circ}, 45^{\circ}\right)$. Side length of plate: $4 \lambda$. Side length of patch: $1 / 8 \lambda$. Polarization of incident field: theta.

These currents are largest at the part of the circumference on which second-order edge diffracted currents are launched. Now consider the $7 \lambda$ results. We see that the currents on the interior part of the plate are smaller than on the $4 \lambda$ plate. The currents on the part of the circumference on which secondorder edge diffracted currents are launched are also smaller than on the $4 \lambda$ plate. This indicates that these currents on the $4 \lambda$ plate are mainly caused by second-order edge diffraction. The currents on that part of the circumference which is close the corner at $x=y=L$ does not decay as the plate size increases. On this part of the edge no second-order edge diffracted currents are launched (see Fig. 5) so these currents must be caused by corner distortion. We see that these corner currents have a complicated behaviour and that is looks as if some interactions between currents launched at the two corners on the edge $y=L$ occur. In order to determine the current caused by corner distortion from one corner we have to suppress these interactions.

\section{Determination of the Current Caused by CORNER Distortion}

This section explains how we can eliminate these interactions by letting the medium that surrounds the plate be lossy and thereby determine the corner current caused by one corner.

A field that propagates in a lossy medium is attenuated and since currents are boundary values of fields they will also be attenuated as they propagate. Therefore, by letting the surrounding medium be lossy we can attenuate currents launched at different points so that they do not interact. Losses can be described by the complex permittivity $\epsilon^{\prime}$ given by

$$
\epsilon^{\prime}=\epsilon_{0}\left(1-j \frac{\sigma}{\omega \epsilon_{0}}\right)
$$

where $\epsilon_{0}$ is the free-space permittivity and $\sigma$ is the conductivity of the medium. We assume that the losses are small,

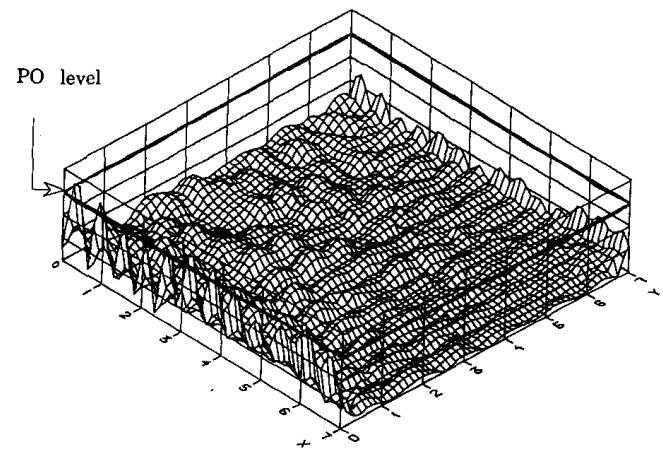

Fig. 7. $x$-component of MM-PO-FW current. Direction of incidence: $\left(\theta^{i}, \phi^{i}\right)=\left(45^{\circ}, 45^{\circ}\right)$. Side length of plate: $7 \lambda$. Side length of patch: $1 / 8 \lambda$. Polarization of incident field: theta.

i.e.,

$$
\frac{\sigma}{\omega \epsilon_{0}} \ll 1
$$

and find that the propagation constant $k^{\prime}$ for the lossy medium is

$$
k^{\prime}=\omega \sqrt{\mu_{0} \epsilon^{\prime}} \simeq\left(2 \pi-j \frac{\pi \sigma}{\omega \epsilon_{0}}\right) \frac{1}{\lambda}
$$

where $\mu_{0}$ is the free-space permeability and $\lambda$ is the free-space wavelength. We define

$$
\beta=\frac{\pi \sigma}{\omega \epsilon_{0}}
$$

and call it the attenuation factor since a plane wave propagating in a $z$-direction decays as $\exp (-\beta z / \lambda)$. We will now explain how the MM-PO-FW current can be calculated when the medium has losses. During these calculations the amplitude of the incident field will be kept constant on the plate, i.e., the incident field on the plate is the same as in the free-space case. Therefore, the physical optics current is the same as in the free-space case. The calculation of the fringe wave currents is in principle more complicated when the surrounding medium is lossy since it requires the solution to the half-plane problem in which the half-plane is situated in a lossy medium. However, we only need to calculate fringe wave currents in the region around the corner $x=y=L$, i.e., close to edges where they are launched. Because the incident field on the plate is the same as in the free-space case, and the fringe wave currents are launched by the incident field at the edges the values of the fringe wave currents close to the edges where they are launched are the same as in the free-space case. Therefore, we can still use the free-space expressions (7), (8) when we calculate the fringe wave current.

For the case with losses in the surrounding medium the complex permittivity $\epsilon^{\prime}$ and the complex propagation constant $k^{\prime}$ in (12) and (14), respectively, are used in the electric-field integral equation instead of the real ones. This is the only change that is made in the moment method program. We do not, therefore, take into account the secondary effects of the currents that are induced in the lossy medium since for small 

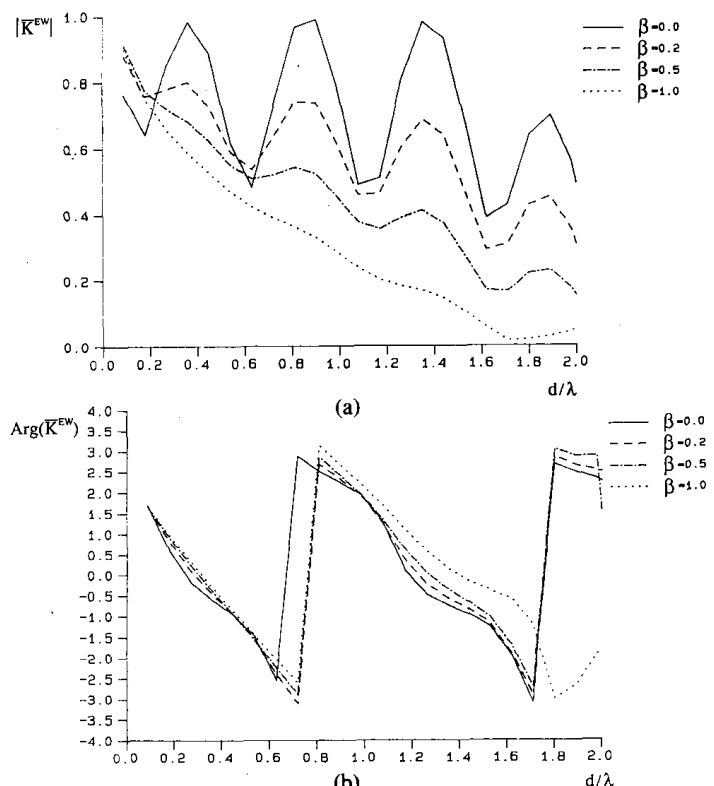

Fig. 8. Amplitude and phase of MM-PO-FW current. Direction of incidence: $\left(\theta^{i}, \phi^{i}\right)=\left(55^{\circ}, 45^{\circ}\right)$. Side length of plate: $3.6 \lambda$. Side length of patch: $1 / 11 \lambda$. Polarization of incident field: theta. (a) Amplitude. (b) Phase.

losses these currents approach a negligible value and thus have negligible effect on the currents in the plate. In other words, the primary effect of the lossy medium is to simply attenuate the surface currents in their directions of propagation. This will be verified later.

We will now show numerical results for MM-PO-FW currents on the plate in the lossy medium. From Figs. 6 and 7 it was seen that it is sufficient to investigate the currents on the patches adjacent to the edge and we will therefore show plots of these currents as functions of the distance $d$ to the corner at $x=y=L$. In the following we use a plate with side length $3.6 \lambda$ and patches with side length $1 / 11 \lambda$. Figs. 8(a) and 8(b) show the amplitude and phase, respectively, of the $x$-component of the MM-PO-FW current along the edge $y=L$ as a function of the distance $d$ to the corner at $x=y=L$, for different attenuation factors in the lossy medium. The incident field is a theta-polarized plane wave, and the direction of incidence is given by $\left(\theta^{i}, \phi^{i}\right)=$ $\left(55^{\circ}, 45^{\circ}\right)$.

From Fig. 8(a) we see that the standing wave pattern seen for $\beta=0$ (free-space) disappears as the amount of loss increases and we get an exponentially decaying current. It should be noted that the amplitude of the incident field is constant on the plate so the decay of the current along the edge is not due to decay of the incident field.

From Fig. 8(b) we see that the phase variation of the current is the phase variation of a free-space wave that travels along the edge, i.e., $\exp (-j 2 \pi d / \lambda)$. It is not the phase variation of the incident field along the edge which for this direction of incidence is $\exp (-j 2 \pi 0.6 d / \lambda)$.

Figs. 8(a) and 8(b) lead therefore to the conclusion that when a wave hits a corner, current waves are launched from the corner and propagate along the edges forming the corner. Such waves are called edge waves because they are guided by edges. Edge waves have been studied before in connection with radiation from dipoles close to edges [15]-[17] and in connection with a semiempirically derived corner diffraction coefficient [18]. In all these references the expressions for the edge waves are given at distances far from the point where they are launched. From our results in the lossy media it is found that the edge wave current approximately decays as $e^{-\beta z / d}$ and thus that the edge wave current in free space close to the corner behaves like

$$
\bar{K}^{\mathrm{EW}}(d)=K_{0} \hat{t} e^{-j k d} .
$$

$K_{0}$ is a constant, $\hat{t}$ an edge tangent unit vector, and $d$ the distance to the corner. It should be emphasized that the current in (16) represents mean values of the actual current on square patches. Therefore, it does not contain singularities since these are integrated out. From the free-space results in Fig. 7 we see that the edge wave current is only significantly nonzero on the patches adjacent to the edge. Therefore, only the current on these patches has been included in the edge wave currents shown in Fig. 8 .

The vector potential for these edge wave currents is thus obtained by integrating the product of the patch length $a$ and the edge wave (EW) current (16) along the edge, i.e.,

$$
\bar{A}(\bar{r})=\frac{\mu e^{-j k r}}{4 \pi r} \int_{\text {edge }} a \bar{K}^{\mathrm{EW}}\left(s^{\prime}\right) e^{j k \hat{r} \cdot \bar{r}^{\prime}} d s^{\prime} .
$$

From (16) and (17) we see that the corner contribution (end point contribution) to the edge wave radiation integral can be found if we know the constant $I=a K_{0}$. From this equation it follows that $K_{0}$ is inversely proportional to the patch size $a$. This is only true when the patch size is so large that edge wave current is significantly nonzero only on the row of patches adjacent to the edge. This is the case for the patch sizes used in this paper. From Figs. 8(a) and 8(b) one finds that the constant $I$ for the theta polarization case with $\left(\theta^{i}, \phi^{i}\right)=\left(55^{\circ}, 45^{\circ}\right)$ is given by

$$
I=e^{j 2.2} 0.09 \lambda \text {. }
$$

The value of $I$ has been calculated for many angles of incidence and for both theta and phi polarizations. The results can conveniently be shown in connection with Fig. 9, where the corner at which the edge wave current is launched is situated at $(x, y, z)=(0,0,0)$ and the edge wave propagates along the negative $x$-axis. The numerical results for $|I|$ in the theta polarization case are shown in Fig. 10 . From these numerical results we see that we can approximate $|I|$ by a polynomial of second degree in $\phi^{i}$. Furthermore, it is found that the phase of $I$ is constant and equal to 2.2 radians for all angles of incidence considered in Fig. 10. In [14] it is shown that these numerical results can be reproduced by the following simple analytical expression:

$$
\begin{aligned}
I_{\theta}\left(\theta^{i}, \phi^{i}\right)= & \lambda e^{j 2.2}\left(A\left(\theta^{i}\right)\left(70^{\circ}-\phi^{i}\right)^{2}\right. \\
& \left.+B\left(\theta^{i}\right)\left(70^{\circ}-\phi^{i}\right)+C\left(\theta^{i}\right)\right)
\end{aligned}
$$




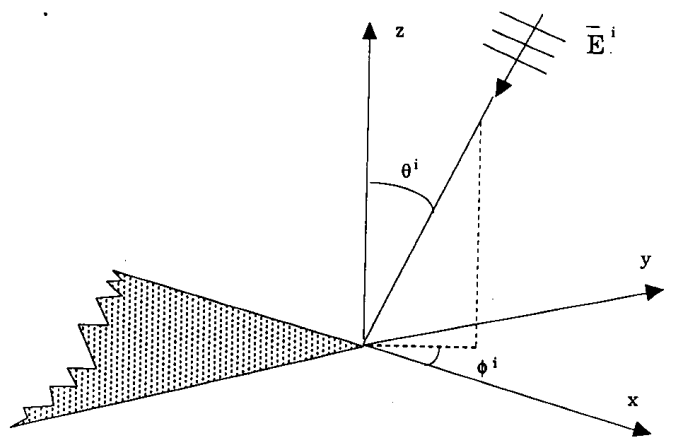

Fig. 9. Scattering configuration.

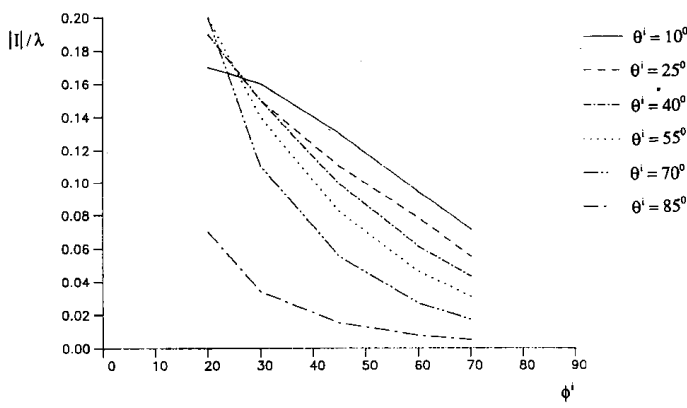

Fig. 10. Numerical results for $|I|$ for theta polarization.

where

$$
\begin{gathered}
A\left(\theta^{i}\right)=A_{0} \cdot\left(\theta^{i}-25^{\circ}\right) \quad \theta^{i} \leq 75^{\circ}, \\
A\left(\theta^{i}\right)=A_{0} \cdot\left(\theta^{i}-25^{\circ}\right)\left(\frac{\left(\cos \left(\theta^{i}\right)\right.}{\cos \left(75^{\circ}\right)}\right)^{1.65}, \quad \theta^{i}>75^{\circ}, \\
B\left(\theta^{i}\right)=B_{0} \cdot\left(90^{\circ}-\theta^{i}\right), \\
C\left(\theta^{i}\right)=C_{0} \cdot\left(90^{\circ}-\theta^{i}\right), \\
A_{0}=1.26 \cdot 10^{-6}, B_{0}=3.48 \cdot 10^{-5}, C_{0}=8.75 \cdot 10^{-4}
\end{gathered}
$$

and the angles of incidence are restricted to

$$
20^{\circ} \leq \phi^{i} \leq 70^{\circ}, \quad 0 \leq \theta^{i} \leq 90^{\circ} .
$$

Let us now consider the special theta polarization case where $\theta^{i}=90^{\circ}$ and $20^{\circ}<\phi^{i}<70^{\circ}$. In this case the total induced surface current on the quarter plane is zero everywhere. From the half-plane solution it follows that the current $\bar{K}^{\mathrm{PO}}+\bar{K}^{\mathrm{FW}}$ also is zero at points where there only exists fringe wave currents launched from one edge. Consequently, the edge wave current along the negative $x$-axis should be zero in this special case. It is seen that the analytical expression in (19) gives this result.

When the incident field is polarized in the phi direction the values of $I$ can be given in a similar analytical expression [14] as

$$
\begin{aligned}
I_{\phi}\left(\theta^{i}, \phi^{i}\right)=\lambda e^{-j 1.0}\left[A\left(\theta^{i}, \phi^{i}\right)\left(\theta^{i}-45^{\circ}\right)^{2}\right. & \\
& \left.+B\left(\phi^{i}\right)\left(\theta^{i}-45^{\circ}\right)+C_{0}\right]
\end{aligned}
$$

where

$$
\begin{gathered}
A\left(\theta^{i}, \phi^{i}\right)=0, \quad \theta^{i}<45^{\circ} \text { or } \phi^{i}>40^{\circ}, \\
A\left(\theta^{i}, \phi^{i}\right)=A_{0}\left(40^{\circ}-\phi^{i}\right)^{1.5}, \theta^{i} \geq 45^{\circ} \text { and } \phi^{i} \leq 40^{\circ} \\
B\left(\phi^{i}\right)=B_{0}\left(90^{\circ}-\phi^{i}\right)^{1.5}, \\
A_{0}=1.00 \cdot 10^{-6}, B_{0}=4.40 \cdot 10^{-6}, C_{0}=1.70 \cdot 10^{-1},
\end{gathered}
$$

and the angles of incidence are restricted to

$$
20^{\circ} \leq \phi^{i} \leq 70^{\circ}, \quad 0 \leq \theta^{i} \leq 90^{\circ} .
$$

We have now obtained analytical expressions from which the asymptotic corner contribution to the edge wave radiation integral can be found. These analytical expressions are only valid for forward directions of incidence. Because of second-order edge diffraction it has not been possible to given expressions for other angles of incidence.

Before we evaluate the asymptotic corner contribution to the radiation integral we will investigate how the current distribution consisting of the physical optics, fringe wave, and edge wave currents compares to the moment method current near a corner in free space. We let the direction of incidence be $\left(\theta^{i}, \phi^{i}\right)=\left(70^{\circ}, 45^{\circ}\right)$ since in this case there is a large region around the corner at $x=y=L$ where no second-order currents are present. Thus, the moment method current around this corner is very much like the current on the quarter plane with tip at $x=y=L$. In Figs. 11(a)-11(c), are shown the amplitude of the $x$-components of the currents $\bar{K}^{\mathrm{PO}}+\bar{K}^{\mathrm{FW}}, \bar{K}^{\mathrm{PO}}+\bar{K}^{\mathrm{FW}}+\bar{K}^{\mathrm{EW}}$, and $\bar{K}^{\mathrm{MM}}$, respectively, for theta polarized incident field. $\bar{K}^{\mathrm{FW}}$ consists of the fringe wave currents launched at the edges $x=L$ and $y=L$, and at these edges a mean value of this current is calculated. $\bar{K}^{\mathrm{EW}}$ consists of the two edge wave currents launched at the corner at $x=y=L$.

From Fig. 11(a) we see that the $\bar{K}^{\mathrm{PO}}+\bar{K}^{\mathrm{FW}}$ current has two lines of discontinuity. These lines are the current boundaries for the fringe wave currents launched at the two edges. Fig. 11(b) shows that when we include the edge wave current we get a standing wave pattern along the edge. This is because the phase variation of the fringe wave and the edge wave currents are different along the edges. The pattern of the moment method current in Fig. 11(c) is qualitatively the same as that of the current in Fig. 11(b) except for some minor ripples. These ripples in the moment method current are caused by the fact that the plate is finite and thus interactions between currents launched at different edges and corners occur. The current in Fig. 11(b) is based on the approximation that the edge wave current does not decay away from the corner. By looking at the moment method current in Fig. 11(c) it is seen that this approximation is very good at distances less than $2 \lambda$ from the corner. From Fig. 11 we conclude that it is a good approximation, for this direction of incidence, to let the corner current be given by the non-decaying edge wave current in (16).

\section{Radiation by a CoRner}

We have now found that for right-angled corners the current due to corner distortion, denoted by $\bar{K}^{c}$ in (1), can 


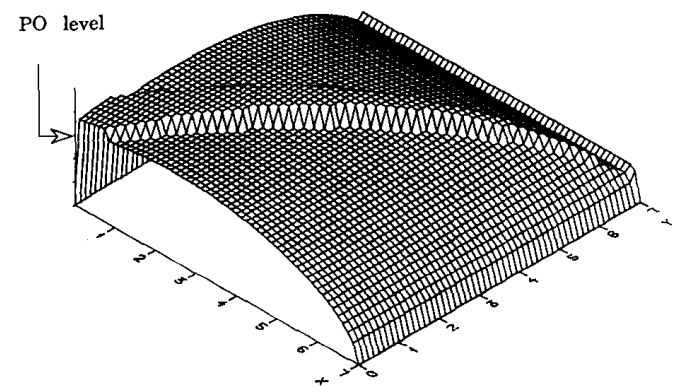

(a)

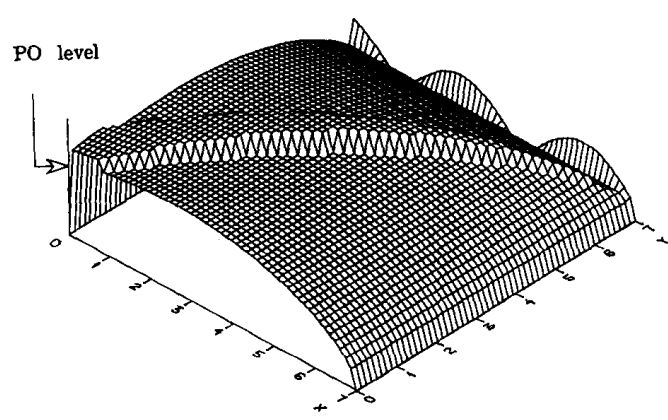

(b)

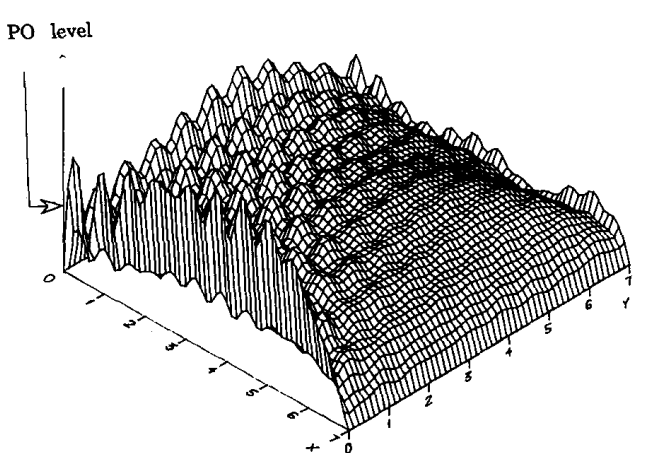

(c)

Fig. 11. Amplitude of $x$-component of current. Direction of incidence: $\left(\theta^{i}, \phi^{i}\right)=\left(70^{\circ}, 45^{\circ}\right)$. Polarization of incident field: theta. (a) Physical optics plus fringe wave currents. (b) Physical optics plus fringe wave plus edge wave currents. (c) Moment method currents.

be described by two edge wave currents launched at the corner. We will now explain how the corner contribution to the radiation integral involving the current distribution (1) can be evaluated analytically. The geometry is shown in Fig. 9, and we restrict ourselves to observation directions where no surface or edge stationary points are close to the corner, i.e., observation directions outside transition regions. Since the current in (1) is divided into three components we get three corner contributions from the radiation integral; namely physical optics, fringe wave, and edge wave contributions [14]. The corner contribution to the physical optics surface radiation integral can be found directly from [19, eq. (8.4.63)] and is

$$
\begin{aligned}
\bar{E}^{\mathrm{PO}}(r)= & \frac{j}{2 \pi k} \sqrt{\frac{\mu_{o}}{\epsilon_{0}}} \frac{1}{\left(\sin \theta \cos \phi+\sin \theta^{i} \cos \phi^{i}\right)} \\
& \cdot \frac{1}{\left(\sin \theta \cos \phi+\sin \theta^{i} \sin \phi^{i}\right)} \\
& \cdot\left[\hat{\theta} \cdot\left(\hat{z} \times \bar{H}^{i}(\bar{o})\right) \hat{\theta}+\hat{\phi} \cdot\left(\hat{z} \times \bar{H}^{i}(\bar{o})\right) \hat{\phi}\right] \frac{e^{-j k r}}{r} .
\end{aligned}
$$

The fringe wave surface radiation integral can be converted into two line radiation integrals by using the equivalent edge currents from [20]. The corner contributions to these two line radiation integrals are easily evaluated by using [19, eq. (3.2.19)]. The corner contribution from the line integral along the negative $x$-axis is

$$
\begin{aligned}
\bar{E}^{\mathrm{FW}}(\bar{r})= & \frac{1}{4 \pi j k} \sqrt{\frac{\mu_{0}}{\epsilon_{o}}}\left\{\hat{r} \times(\hat{r} \times \hat{x})\left[\sqrt{\frac{\epsilon_{0}}{\mu_{0}}} \bar{E}^{i}(\bar{o}) \cdot \hat{x}\right.\right. \\
& \cdot D_{E}^{I}\left(\beta_{x}^{i}, \phi_{x}^{i}, \beta_{x}, \phi_{x}\right)+\bar{H}^{i}(\bar{o}) \\
& \left.\cdot \hat{x} D_{H}^{I}\left(\beta_{x}^{i}, \phi_{x}^{i}, \beta_{x}, \phi_{x}\right)\right] \\
& \left.+\hat{r} \times \hat{x} \bar{H}^{i}(\bar{o}) \cdot \hat{x} D_{H}^{M}\left(\beta_{x}^{i}, \phi_{x}^{i}, \beta_{x}, \phi_{x}\right)\right\} \\
& \cdot \frac{1}{\sin \theta \cos \phi+\sin \theta^{i} \cos \phi^{i}} \cdot \frac{e^{-j k r}}{r}
\end{aligned}
$$

The expression for the corner contribution from the radiation integral along the negative $y$-axis is (22) with $x$ replaced by $y$ and cos replaced by sin.

The parameters in these fringe wave corner contribution expressions are as follows:

$$
\begin{aligned}
& D_{E}^{I}\left(\beta^{i}, \phi^{i}, \beta, \phi\right)= \frac{2 \sqrt{2}}{\sin ^{2} \beta^{i}} \frac{\sin \frac{\phi^{i}}{2}}{\cos \phi^{i}+\mu} \\
& \cdot\left[\sqrt{2} \cos \frac{\phi^{i}}{2}-\sqrt{1-\mu}\right], \\
& D_{H}^{I}\left(\beta^{i}, \phi^{i}, \beta, \phi\right)= \frac{2}{\sin \beta^{i}} \frac{1}{\cos \phi^{i}+\mu} \\
& \cdot\left[\frac{\cot \beta \cos \phi-\mu \cot \beta^{i}}{\sqrt{1-\mu}}\right. \\
& \cdot \sqrt{2} \cos \frac{\phi^{i}}{2}-\cot \beta \cos \phi \\
&\left.-\cot \beta^{i} \cos \phi^{i}\right] \\
& D_{H}^{M}\left(\beta^{i}, \phi^{i}, \beta, \phi\right)= \frac{2 \sin \phi}{\sin \beta \sin \beta^{i}} \\
& \cdot \frac{1}{\cos \phi^{i}+\mu}\left[\frac{\sqrt{2} \cos \frac{\phi^{i}}{2}}{\sqrt{1-\mu}}-1\right]
\end{aligned}
$$




$$
\begin{gathered}
\mu=\frac{\sin \beta^{i} \sin \beta \cos \phi+\cos \beta^{i} \cos \beta-\cos ^{2} \beta^{i}}{\sin ^{2} \beta^{i}} \\
\beta_{x}^{i}=\arccos \left(-\sin \theta^{i} \cos \phi^{i}\right) \\
\phi_{x}^{i}=2 \pi-\arccos \left(\frac{-\sin \theta^{i} \sin \phi^{i}}{\sqrt{1-\sin ^{2} \theta \cos ^{2} \phi^{i}}}\right) \\
\beta_{x}=\arccos (\sin \theta \cos \phi) \\
\phi_{x}=2 \pi-\arccos \left(\frac{-\sin \theta \sin \phi}{\sqrt{1-\sin { }^{2} \theta \cos ^{2} \phi}}\right) \\
\beta_{y}^{i}=\arccos \left(-\sin \theta^{i} \sin \phi^{i}\right) \\
\phi_{y}^{i}=\arccos \left(\frac{-\sin \theta^{i} \cos \phi^{i}}{\sqrt{1-\sin ^{2} \theta^{i} \sin ^{2} \phi^{i}}}\right) \\
\beta_{y}=\arccos (\sin \theta \sin \phi)
\end{gathered}
$$

and

$$
\phi_{y}=\arccos \left(\frac{-\sin \theta \cos \phi}{\sqrt{1-\sin ^{2} \theta \sin ^{2} \phi}}\right) .
$$

The evaluation of the end point contribution from the physical optics and fringe wave currents has previously been performed in [21] and [22].

The corner contribution from the edge wave radiation integral (17) is also easily evaluated by using [19, eq. (3.2.19)] and the result is

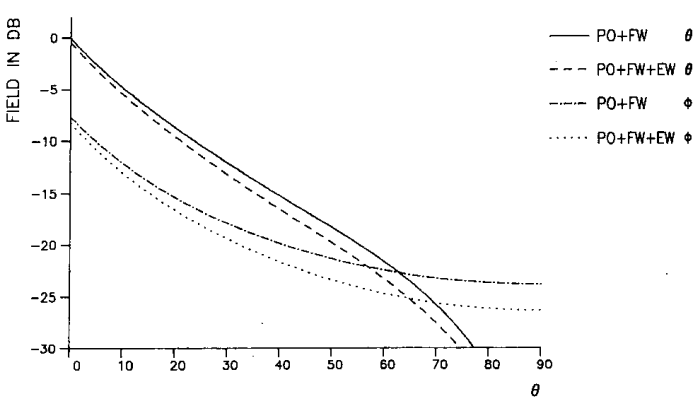

Fig. 12. Theta and phi components of electric field. Direction of incidence: $\left(\theta^{i}, \phi^{i}\right)=\left(40^{\circ}, 20^{\circ}\right)$. Direction of observation: $\phi=45^{\circ}$. Polarization of incident field: theta.

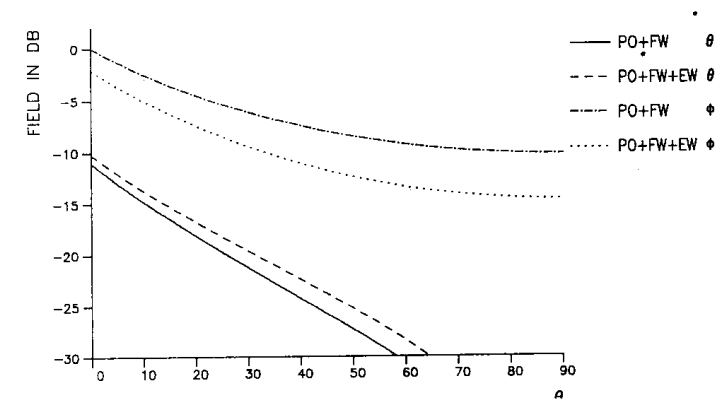

Fig. 13. Theta and phi components of electric field. Direction of incidence: $\left(\theta^{i}, \phi^{i}\right)=\left(70^{\circ}, 30^{\circ}\right)$. Direction of observation: $\phi=45^{\circ}$. Polarization of incident field: phi.

$$
\begin{aligned}
\bar{E}^{\mathrm{EW}}(\bar{r})= & \frac{-1}{4 \pi}\left\{\frac{\bar{E}^{i}(\bar{o}) \cdot \hat{\theta}^{i} I_{\theta}\left(\theta^{i}, \phi^{i}\right)+\bar{E}^{i}(\bar{o}) \cdot \hat{\phi}^{i} I_{\phi}\left(\theta^{i}, \phi^{i}\right)}{1+\sin \theta \cos \phi}\right. \\
& \cdot(\cos \theta \cos \phi \hat{\theta}-\sin \phi \hat{\phi}) \\
& +\frac{\bar{E}^{i}(\bar{o}) \cdot \hat{\theta}^{i} I_{\theta}\left(\theta^{i}, 90^{\circ}-\phi^{i}\right)-\bar{E}^{i}(\bar{o}) \cdot \hat{\phi}^{i} I_{\phi}\left(\theta^{i}, 90^{\circ}-\phi^{i}\right)}{1+\sin \theta \sin \phi} \\
& \cdot(\cos \theta \sin \phi \hat{\alpha}-\cos \phi \hat{\phi})\} \frac{e^{-j k r}}{r}
\end{aligned}
$$

where $I_{\theta}(\theta, \phi)$ and $I_{\phi}(\theta, \phi)$ are given in (19) and (20), respectively. The corner diffraction coefficients are identified from (21), (22), and (35) by noting that the phase factor is $e^{-j k r}$ and the divergence factor is $1 / r$.

We will now show some numerical results for corner diffracted fields and investigate if it is important to include the edge wave currents. Again the geometry is shown in Fig. 9 and we calculate the scattered electric field in the half-plane $\phi=45^{\circ}$ as a function of the angle $\theta$. Zero $\mathrm{dB}$ corresponds to the maximum of the theta and phi components of the electric field scattered by the current $\bar{K}^{\mathrm{PO}}+\bar{K}^{\mathrm{FW}}$. First consider the case where the incident field is polarized in the theta direction and the direction of incidence is given by $\left(\theta^{i}, \phi^{i}\right)=$ $\left(40^{\circ}, 20^{\circ}\right)$. In this case both theta and phi components of the electric field scattered by the current $\bar{K}^{\mathrm{PO}}+K^{\mathrm{FW}}$, and the current $K^{\mathrm{PO}}+\bar{K}^{\mathrm{FW}}+\bar{K}^{\mathrm{EW}}$ are shown in Fig. 12.

We see that there is a maximum difference of about 2 and
$3 \mathrm{~dB}$ between the theta and phi components, respectively, calculated from $\bar{K}^{\mathrm{PO}}+\bar{K}^{\mathrm{FW}}$ and $\bar{K}^{\mathrm{PO}}+\bar{K}^{\mathrm{FW}}+\bar{K}^{\mathrm{EW}}$. Fig. 13 shows results for the case where the incident field is polarized in the phi direction and the direction of incidence is given by $\left(\theta^{i}, \phi^{i}\right)=\left(70^{\circ}, 40^{\circ}\right)$.

In this case the maximum difference between the theta components is about $3 \mathrm{~dB}$ and the maximum difference between the phi components is $4 \mathrm{~dB}$. We therefore conclude that it is important to include the current caused by corner distortion in the calculation of corner diffracted fields. Of course, corner diffracted fields are usually negligible in observation directions where rays from surfaces or rays along cones of diffraction from edges also exist. However, there are large regions of space for most scatterers where no surface or edge stationary points exist and thus only corner diffracted fields are presented [23].

It should be noted that this corner diffraction coefficient, at 
its present stage, works only for forward directions of incidence and applies only to flat plates with right-angled corners. Therefore, the work presented in this paper is an initial, though important step toward a useful, accurate corner diffraction coefficient that is valid for all angles of incidence.

\section{CONCLUSION}

The induced surface current density near a right-angled corner on a perfectly conducting flat scatterer, illuminated by a plane wave, has been investigated. This current was written as the sum of the physical optics current, the fringe wave current, and the corner current. Each of these currents accounts for different diffraction processes on the scatterer. The physical optics current accounts for the surface effect, and the fringe wave current, which was found from the half-plane solution, accounts for the distortion caused by the edges. The corner current accounts for the distortion of the current caused by the corner. The corner current was found from a numerical solution to the electric-field integral equation for a square plate. In order to find the corner current caused by one corner it was necessary to insert a loss into the medium surrounding the plate. It was found that the corner current for the right-angled corner, for forward directions of incidence, to a good approximation consisted of two edge wave currents. These edge wave currents were launched at the corner and propagated along the two edges forming the corner. Simple analytical expressions for the edge wave currents as a function of the direction of incidence were constructed from the numerical results.

The field scattered by the corner was calculated as the asymptotic corner contribution to the radiation integral involving the approximate current. It was found that the corner current is important in the calculation of the corner diffracted field. In particular, the field calculated by including the corner current differed by as much as $4 \mathrm{~dB}$ from the field obtained by not including the corner current. Further calculations have shown differences as large as $7 \mathrm{~dB}$ [14].

In this paper we have given expressions for the corner current caused by a right-angled corner for forward directions of incidence. Expressions outside this region are not directly obtainable from the square plate results because of second-order edge diffraction. Furthermore, for small-angled corners it may not be possible for any angles of incidence to describe the corner current by two edge wave currents alone. Therefore much work still has to be done in order to determine corner currents for a general corner illuminated from an arbitrary direction.

\section{ACKNOWLEDGMENT}

The author wishes to thank Jorgen Appel-Hansen, for suggesting and encouraging the work, Olav Breinbjerg for providing the program to calculate the half-plane fringe wave currents, and Arthur D. Yaghjian for many helpful discussions.

\section{REFERENCES}

[1] R. S. Satterwhite, "Diffraction by a plane angular sector," Ph.D. dissertation, Ohio State Univ., Columbus, OH, 1969.

[2] T. B. Hansen, "Diffraction by a plane angular sector, a new deriva- tion," IEEE Trans. Antennas Propagat., vol. 38, pp. 1892-1894, Nov. 1990.

[3] R. S. Satterwhite, "Diffraction by a quarter plane, the exact solution, and some numerical results," IEEE Trans. Antennas Propagat., vol. AP-22, pp. 500-503, May 1974.

[4] L. B. Felsen and N. Marcuvitz, Radiation and Scattering of Waves. Englewood Cliffs, NJ: Prentice-Hall, 1973.

[5] T. B. Hansen, "An attempt to obtain a useful representation for field scattered by a plane angular sector when both source and field points are far from the corner," Electromagn. Inst., Tech. Univ. Denmark, R 426, Mar. 1990.

[6] P. M. Morse and H. Feshbach, Methods of Theoretical Physics, vol. 1. New York: McGraw-Hill, 1953.

[7] V. P. Smyshlyaev, "On the diffraction of waves by cones at high frequencies," LOMI, preprint E-9-89, Leningrad, U.S.S.R., 1989.

[8] _-, "Diffraction of electromagnetic waves by conical obstacles in high frequencies," in Proc. Int. URSI Symp. Electromagn. Wave Theory, Stockholm, Sweden, Aug. 1989.

[9] -, "Diffraction by conical surfaces at high frequencies," Wave Motion, vol. 12, pp. 329-339, June 1990.

[10] J. J. Bowman et al., Electromagnetic and Acoustic Scattering by Simple Shapes. Amsterdam: North Holland, 1969.

[11] O. Breinbjerg, "Equivalent edge current analysis of electromagnetic scattering by plane structures," Ph.D. dissertation, Electromagn. Inst., Tech. Univ. Denmark, LD 83, 1991.

[12] A. W. Glisson and D. R. Wilton, "Simple and efficient numerical methods for problems of electromagnetic radiation and scattering from surfaces," IEEE Trans. Antennas Propagat., vol. AP-28, pp. 593-603, Sept. 1980.

[13] T. K. Sarkar and E. Arvas, "On a class of finite step iterative methods (conjugate directions) for the solution of an operator equation arising in electromagnetics," IEEE Trans. Antennas Propagat., vol. AP-33, pp. 1058-1066, Oct. 1985.

[14] T. B. Hansen, "Diffraction of electromagnetic waves by corners on perfect conductors," Ph.D. dissertation, Electromagn. Inst., Tech. Univ. Denmark, LD 86, 1991.

[15] O. M. Buyukdura, "Radiation from sources and scatterers near the edge of a perfectly conducting wedge," Ph.D. dissertation, Ohio State Univ., Columbus, OH, 1984

[16] L. W. Pearson, "The electromagnetic edge wave due to a point source of current radiating in the presence of a conducting wedge," IEEE Trans. Antennas Propagat., vol. AP-34, pp. 1125-1132, Sept. 1985

[17] L. P. Ivrissimtzis and R. J. Marhefka, "Edge wave vertex and edge diffraction," Radio Sci., vol. 24, pp. 771-784, Nov.-Dec. 1989.

[18] F. A. Sikta, "UTD analysis of electromagnetic scattering by flat plate structures," Ph.D. dissertation, Ohio State Univ., Columbus, OH 1984.

[19] N. Bleistein and R. A. Handelsman, Asymptotic Expansions of Integrals.' New York: Dover, 1986.

[20] A. Michaeli, "Elimination of infinities in equivalent edge currents, Part I: Fringe current components," IEEE Trans. Antennas Propagat., vol. AP-34, pp. 912-918, July 1986.

[21] J. B. Keller, R. M. Lewis, and B. D. Seckler, "Diffraction by an aperture. II," J. Appl. Phys., vol. 28, pp. 570-579, May 1957.

[22] T. J. Brinkley and R. J. Marhefka, "Far zone bistatic scattering from flat plates," ElectroSci. Lab., Ohio State Univ., Columbus, $\mathrm{OH}$ Tech. Rep. 718295-8, June 1988.

[23] M. G. Cote, M. B. Woodworth, and A. D. Yaghjian, "Scattering from the perfectly conducting cube," IEEE Trans. Antennas Propagat., vol. 36, pp. 1321-1329, Sept. 1988.

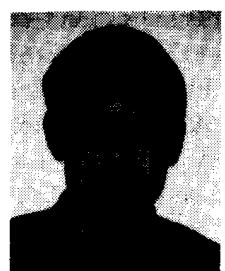

Thorkild B. Hansen (S'91) was born in Odense, Denmark, on January 26, 1965. He received the M.S.E.E. degree from the Technical University of Denmark, Lyngby, Denmark, in 1989, where he has been pursuing the Ph.D. degree in electrical engineering since 1989 .

In the summer of 1990 he was a Visiting Research Scientist at the Electromagnetics Directorate of Rome Air Development Center, Hanscom Air Force Base, MA. His research interests include high frequency techniques. 